\title{
COORDINATES OF THE REPRESENTATION SPACE IN THE SEMISIMPLE LIE GROUP OF RANK ONE
}

\author{
INKANG KIM
}

\begin{abstract}
In this paper we show that the space of irreducible representations from a finitely presented group into the group of isometries of a rank one symmetric space of noncompact type, embeds into $\mathbb{R}^{n}$ for some $n$, where the coordinates are the translation lengths of isometries in the representation. The ingredients of the proof consist of the two facts that the representation is determined by its marked length spectrum and that the nested sequence of algebraic subvarieties is stabilised at a finite step by the Noetherian property of the polynomial ring. As a minor application, we use this fact to simplify McMullen's proof about the exponential algebraic convergence of Thurston's double limit to the geometrically infinite manifold in the space of discrete faithful representations of $\pi_{1}(S)$ in $\operatorname{Iso}^{+}\left(H_{\mathbb{R}}^{3}\right)$.
\end{abstract}

\section{INTRODUCTION}

It is known that the lengths of $9 g-9$ closed geodesics of the Riemann surface $S$ of genus $g$, determine the hyperbolic structure of the surface in the Teichmüller space [2]. So there is a smooth map from the Teichmüller space of the genus- $g$ surface into $\mathbb{R}^{9 g-9}$ where a point $\rho$ corresponds to the point $\left(l_{\rho}\left(g_{1}\right), \cdots, l_{\rho}\left(g_{9_{g}-9}\right)\right)$. Here $l_{\rho}(g)$ is the length in the $\rho$-metric of the closed geodesic in the homotopy class of $g \in \pi_{1}(S)$.

It was not known that such a result holds for higher dimensional cases. In this paper we answer the question affirmatively for all dimensions of rank one symmetric spaces of noncompact type. The theorem is much more general than the question, namely such a parameterisation is true even for the space $\Re^{0}$ of non-elementary irreducible representations.

The translation length of an isometry is a minimum distance it moves. The marked length spectrum of a representation is a function associating the translation length of the isometry $\rho(g)$, to each $g$ in the group. See Section 3 for definitions.

The idea of the proof is very simple. By [4], it is known that an irreducible representation into the group of isometries of hyperbolic $n$-space is determined up to conjugacy

Received 23rd March, 1998

I want to thank C.T. McMullen for asking the question about our main theorem and many useful suggestions. Also I want to thank A. Casson for his help as an adviser.

Copyright Clearance Centre, Inc. Serial-fee code: 0004-9729/98 \$A2.00+0.00. 
by its marked length spectrum. So the task is to choose a finite set of elements of the group such that the marked length spectrum on this set determines the representation up to conjugacy. This can be done using the Noetherian property of the polynomial ring. More precisely we find some polynomial equations defining the subvariety of $\Re \times \Re$, which consists of pairs of representations having the same marked length spectrum on the finite set of elements in the group, using the Lorentzian model of hyperbolic space. As we increase the number of elements of the group, the number of subvarieties defined by these elements decreases. But this should be stable after a finite number of steps by the Noetherian property of the polynomial ring. We can state the main theorem in the algebraic point of view as follows.

THEOREM. Let $R^{0}(G)$ be the space of irreducible representations from a finitely presented group $G$ into Iso $(X)$. Then there is a finite set of elements, $g_{1}, \cdots, g_{N}$ such that if $l\left(\rho\left(g_{i}\right)\right)=l\left(\phi\left(g_{i}\right)\right)$ for $i=1, \cdots, N$, then they are conjugate, and so $\chi_{\rho}=\chi_{\phi}$ for any $\rho, \phi \in R^{0}(G)$ where $\chi_{\phi}$ is the character of $\phi$ when Iso $(X)$ is identified with some subgroup in $G L_{n}(\mathbb{F})$ where $\mathbb{F}=\mathbb{R}$ or $\mathbb{C}$.

\section{The SPACE of Representations: Algebraic Variety}

Let $G=\left\langle g_{i}, i=1, \cdots, n ; r_{1}=\cdots=r_{k}=1\right\rangle$. The space of representations $R(G)$ from $\mathrm{G}$ to $G L_{m}(\mathbb{F})$ is a set of homomorphisms from $\mathrm{G}$ into $G L_{m}(\mathbb{F})$. It is the set of points $\left(\rho\left(g_{1}\right), \cdots, \rho\left(g_{n}\right)\right)$ in $G L_{m}(\mathbb{F})^{n} \subset \mathbb{F}^{m^{2} \times n}$ which satisfy $\rho\left(r_{i}\right)=1$. Then it is easy to see that $R(G)$ is an algebraic variety. In this paper we are particularly interested when representations are in Iso $\left(H_{\mathbf{F}}^{n}\right)$. The Lie group Iso $\left(H_{\mathbf{F}}^{n}\right)$ acts on $R(G)$ by conjugation. A representation is irreducible if it does not leave invariant any totally geodesic subspace except $H_{\mathbf{F}}^{n}$. Let $R^{0}(G)$ be the set of irreducible representations. Then the action of Iso $\left(H_{\mathbf{F}}^{n}\right)$ is free on $R^{0}(G)$ since $\alpha \rho \alpha^{-1}=\rho$ for some $\alpha \neq 1 \in$ Iso $\left(H_{\mathbb{F}}^{n}\right)$ only if $\rho$ is reducible and $\alpha$ is an identity on the totally geodesic subspace which $\rho$ leaves invariant. Furthermore $R^{0}(G)$ is open since a small perturbation of an irreducible representation remains as an irreducible representation. So $\Re^{0}(G)=R^{0}(G)$ / Iso $\left(H_{\mathrm{F}}^{n}\right)$ is a smooth open set in $\Re(G)=R(G) /$ Iso $\left(H_{\mathbf{F}}^{\mathfrak{n}}\right)$. See $[\mathbf{3}, \mathbf{1}]$ for references. In this paper we want to find a smooth parametrisation of $\Re(G)^{0}$ by the translation lengths of the finite set of isometries.

\section{ISOMETRIES OF HYPERBOLIC SPACE AND THE MARKED LENGTH SPECTRUM}

The translation length of an isometry $\alpha$ acting on a hyperbolic space $H$ equipped with a hyperbolic metric $d$, is defined as:

$$
l(\alpha)=\inf _{x \in H}\{d(x, \alpha(x))\} .
$$

There are three kinds of isometries in hyperbolic space. When $l(\alpha)=0$ and it is realised in the space, $\alpha$ is called elliptic. When $l(\alpha)=0$ but it is not realised in the space, $\alpha$ is called parabolic. Finally when $l(\alpha)>0, \alpha$ is called hyperbolic. 
When we identify Iso $\left(H_{\mathbf{F}}^{n}\right)$ with a component of $O_{\mathbf{F}}(n, 1)$ when $\mathbb{F}=\mathbb{C}$ or $\mathbb{R}$, the trace of a parabolic element is $n+1$ and the trace of an elliptic or a hyperbolic element is $2 \sum \cos \theta_{i} \pm \varepsilon+2 \cosh r, \varepsilon=0,1$ for real hyperbolic, $\sum e^{i \theta_{j}}+2 \cosh r$ for complex hyperbolic, where $r$ is the translation length of the isometry.

DEFINITION 1. Two representations $\rho, \phi: G \rightarrow$ Iso $(H)$ have the same marked length spectrum if and only if $l(\rho(g))=l(\phi(g))$ for all $g \in G$.

A representation is non-elementary if the image under the representation is nonelementary, that is, its limit set is infinite. Here is the theorem that we need in this paper.

THEOREM 1. Let $\rho, \phi$ be non-elementary, irreducible representations from a group $G$ into Iso $(X)$ where $X$ is a rank one symmetric space of non-compact type. If they have the same marked length spectrum, then they are conjugate.

The proof is quite long and needs understanding of the sub-Riemannian nature of the ideal boundary of rank one symmetric spaces. Since it is a separate issue, we just refer the readers to [5].

\section{RING OF SYMMETRIC FUNCTIONS}

The subring of symmetric functions in a polynomial ring is finitely generated. A well-known basis of the ring is the set of elementary symmetric functions:

$$
\begin{gathered}
s_{1}=\sum_{i=1}^{n} x_{i} ; \\
s_{2}=\sum_{\substack{1 \leqslant i<j \leqslant n \\
\cdots}} x_{i} x_{j} ; \\
s_{n}=x_{1} x_{2} \cdots x_{n} .
\end{gathered}
$$

To introduce another well-known basis, we need the following concepts. A partition $\lambda$ of $n$ is any finite sequence of non-negative integers in decreasing order: $\lambda=\left(\lambda_{1}, \cdots, \lambda_{k}\right)$ such that $\sum \lambda_{i}=n$. Let

$$
P_{k}\left(x_{1}, \cdots, x_{n}\right)=\sum_{i=1}^{n} x_{i}^{k}
$$

Define $P_{\lambda}=\Pi P_{\lambda_{i}}$ for a partition $\lambda=\left(\lambda_{1}, \cdots, \lambda_{k}\right)$. It is known that the set of symmetric functions of the degree $n$ is generated by $P_{\lambda}$ as a vector space, where $\lambda$ is a partition of $n$. For example when $n=3$, a basis of the degree-3 symmetric functions is $P_{1}^{3}, P_{2} P_{1}, P_{3}$. So the ring of symmetric functions of degree up to $n$ is generated by $P_{1}, \cdots, P_{n}$.

The following lemma is elementary, yet important to define the algebraic subvarieties in our later discussion. 
Lemma 1. For any positive integer $n, P_{n+1}\left(x_{1}, \cdots, x_{n}\right)$ can be expressed as some polynomial in $P_{i}\left(x_{1}, \cdots, x_{n}\right)$ for $i=1, \cdots, n$.

ProOF: Consider the ring of symmetric functions in variables $x_{1}, \cdots, x_{n}$. Then the elementary symmetric function $s_{n+1}$ is equal to zero since there are only $n$ variables. Yet $s_{n+1}$ can be expressed as the linear combination of $P_{\lambda}$ where $\lambda$ are partitions of $n+1$. This implies that $P_{n+1}$ can be expressed as a polynomial in $P_{k}$ where $k=1, \cdots, n$.

\section{Parametrisation of the SPACE OF IRREDUCible REPRESENTATIONS By a FINITE SET OF TRANSLATION LENGTHS}

Lemma 2. Let $A$ be a hyperbolic isometry in Iso $\left(H_{\mathbf{F}}^{n}\right)$ where $\mathbb{F}=\mathbb{R}$ or $\mathbb{C}$. Then there is a polynomial relation among $\operatorname{tr} A^{k}$ and $\cosh r$ where $r$ is the translation length of a hyperbolic isometry.

PROOF: If we identify Iso $\left(H_{\mathbb{P}}^{n}\right)$ with a component of $O_{\mathbb{F}}(n, 1)$ in the Lorentzian model, then by conjugating the isometry, we can put $A$ into the form:

$$
\left[\begin{array}{cccccc}
\cos \theta_{1} & \sin \theta_{1} & 0 & 0 & 0 & 0 \\
-\sin \theta_{1} & \cos \theta_{1} & \cdots & 0 & 0 & 0 \\
0 & 0 & 0 & \pm 1 & 0 & 0 \\
0 & 0 & 0 & \cdots & 0 & 0 \\
0 & 0 & 0 & 0 & \cosh r & \sinh r \\
0 & 0 & 0 & 0 & \sinh r & \cosh r
\end{array}\right]
$$

in real hyperbolic case. In complex hyperbolic case, $A$ can be conjugated into the form:

$$
\left[\begin{array}{ccccc}
e^{i \theta_{1}} & 0 & 0 & 0 & 0 \\
0 & \cdots & 0 & 0 & 0 \\
0 & 0 & 0 & \cosh r & \sinh r \\
0 & 0 & 0 & \sinh r & \cosh r
\end{array}\right] .
$$

Then

$$
\operatorname{tr} A^{k}=\sum_{i} \cos k \theta_{i}+2 \cosh k r
$$

for real hyperbolic, and

$$
\operatorname{tr} A^{k}=\sum_{i} e^{k i \theta_{i}}+2 \cosh k r
$$

for complex hyperbolic. Since $\cos k \theta$ can be expressed in terms of $\cos ^{m} \theta$ for $m \leqslant k$, we get

$$
\sum_{i} \cos ^{k} \theta_{i}=P_{k}\left(\operatorname{tr} A^{m}, \cosh m r\right)
$$

for some polynomial $P_{k}$ and $m=1, \cdots, k$ for the real hyperbolic space. Then by Lemma 1 , there is a polynomial $P$ such that $P\left(\operatorname{tr} A^{m}, \cosh m r\right)=0,1 \leqslant m \leqslant[(n-1) / 2]+1$. For the complex hyperbolic case, Lemma 1 can be used directly. 
Now we are ready to prove the main theorem (for both real and complex cases).

THEOREM 2. Let $\Re^{0}(G)$ be the space of irreducible representations, up to conjugacy, from a finitely presented group $G$ into Iso $(X)$ where $X=H_{\mathbf{R}}^{n}$ or $H_{\mathbb{C}}^{n}$. Then there is a smooth embedding $f$ from $\Re^{0}(G)$ into $\mathbb{R}^{k}$ and $g_{1}, \cdots, g_{k} \in G$ for some $k$, such that $f(\phi)=\left(l\left(\phi\left(g_{1}\right)\right), \cdots, l\left(\phi\left(g_{k}\right)\right)\right)$ for $\phi \in \Re^{0}(G)$.

Proof: From Section 2, we know $R(G) \times R(G)$ is an algebraic variety. Then for any given finite set $S_{k}=\left\{g_{1}, \cdots, g_{k}\right\} \subset G$, the set $V_{k}$ consisting of pairs of representations which have the same marked length spectrum on $S_{k}$, is an algebraic subvariety by Lemma 2 since if $\rho$ and $\phi$ have the same marked length spectrum on $S_{k}$, there are polynomials $P_{i}$ and $P_{i}^{\prime}$ such that $P_{i}\left(\operatorname{tr} \rho\left(g_{i}^{m}\right), \cosh m r_{i}\right)=0=P_{i}^{\prime}\left(\operatorname{tr} \phi\left(g_{i}^{m}\right), \cosh m r_{i}\right)$ for $1 \leqslant i \leqslant k$. As we increase the sets $S_{k} \subset S_{k+1}$ to exhaust $G$, the corresponding algebraic subvarieties $V_{k}$ are decreasing but by the Noetherian property of the polynomial ring, $V_{N}=V_{\infty}$ for some positive integer $N$. Let $\mathcal{V}_{k}^{0}=\left\{V_{k} \cap\left(R^{0}(G) \times R^{0}(G)\right)\right\} /\left(\right.$ Iso $\left(H_{\mathbb{F}}^{n}\right) \times$ Iso $\left.\left(H_{\mathbb{F}}^{n}\right)\right)$. By Theorem 1 saying that an irreducible representation is determined up to conjugacy by its marked length spectrum, $\mathcal{V}_{\infty}^{0}$ is the subset of a diagonal of $\Re^{0}(G) \times \Re^{0}(G)$. So we conclude that there is a finite set of elements $g_{1}, \cdots, g_{N} \in G$ such that an irreducible representation is determined up to conjugacy by its marked length spectrum on this set.

Since the function $l_{g}: \Re^{0}(G) \rightarrow \mathbb{R}$, defined by $l_{g}(\phi)=l(\phi(g))$ is a smooth function, the map $f$ is smooth.

It is worthwhile to mention the following corollary, in dimension 2 and 3.

CoRollary 1. There is an embedding which is smooth except at the reducible representations, from the space of non-elementary representations from a finitely presented group into the group of isometries of real hyperbolic 2 or 3 space, to $\mathbb{R}^{k}$ where the mapping is given by the traislation lengtiss of some $k$ elements in the group.

PRoOF: It follows from the fact that in dimension 2 or 3 , we do not need irreducibility of a representation to conclude that the representation is determined by its marked length spectrum. See [4] or [5].

In 3-dimensional topology, it is important to determine when two homotopy equivalent 3-manifolds are homeomorphic. Corollary 1 in turn implies the following corollary.

Corollary 2. Let $M, N$ be two hyperbolic 3-manifolds which are homotopy equivalent. $M$ and $N$ are isometric, so homeomorphic, if the lengths of closed geodesics of some finite set of conjugacy classes in $\pi_{1}(M)=\pi_{1}(N)$ are the same.

\section{Quaternionic and Cayley hyPERbolic CASES}

Since quaternions and Cayley numbers are not commutative, most of the properties which hold in the real or complex field break down. But we want to prove that Theorem 2 holds in the quaternionic ring. For the Cayley hyperbolic case, the calculation is very complicated and we leave the details for future work. 
There is a natural way to identify quaternions with a pair of complex numbers. Since $x_{0}+x_{1} i+x_{2} j+x_{3} k=x_{0}+x_{1} i+\left(x_{2}+x_{3} i\right) j, x_{0}+x_{1} i+x_{2} j+x_{3} k$ can be identified with $\left(x_{0}+x_{1} i, x_{2}+x_{3} i\right)$. So the $n$-dimensional quaternions $\mathbb{H}^{n}$ can be identified with $\mathbb{C}^{2 n}$. By this identification there is a group monomorphism sending a matrix $A+B j$ in $G L_{n}(\mathbb{H})$ acting on $\left(x_{1}+y_{1} j, \cdots, x_{n}+y_{n} j\right)$ on the right, to the matrix in $G L_{2 n}(\mathbb{C})$

$$
\left[\begin{array}{cc}
A & B \\
-\bar{B} & \bar{A}
\end{array}\right]
$$

which acts on $\left(x_{1}, \cdots, x_{n} ; y_{1}, \cdots, y_{n}\right)$ on the right. Then $S p(n, 1)$ can be identified with $U(n, 1 ; n, 1)$ which preserves the $(n, 1 ; n, 1)$ symplectic form, that is, the form

$$
\begin{gathered}
\left\langle\left(x_{1}^{1}, \cdots, x_{1}^{n+1} ; y_{1}^{1}, \cdots, y_{1}^{n+1}\right),\left(x_{2}^{1}, \cdots, x_{2}^{n+1} ; y_{2}^{1}, \cdots, y_{2}^{n+1}\right)\right\rangle \\
=\left(\sum_{l=1}^{n} y_{1}^{l} x_{2}^{l}-x_{1}^{l} y_{2}^{l}\right)-\left(y_{1}^{n+1} x_{2}^{n+1}-x_{1}^{n+1} y_{2}^{n+1}\right) .
\end{gathered}
$$

Note the trace of $A+B j$ is not invariant under conjugation, but $\operatorname{Re}(\operatorname{tr}(A+B j))$ is invariant under conjugation since the trace of

$$
\left[\begin{array}{cc}
A & B \\
-\bar{B} & \bar{A}
\end{array}\right]
$$

is $2 R e(\operatorname{tr} A)=2 \operatorname{Re}(\operatorname{tr}(A+B j))$.

THEOREM 3. Let $\Re^{0}(G)$ be the space of irreducible representations, up to conjugacy, from a finitely presented group $G$ into Iso $\left(H_{\mathrm{HH}}^{n}\right)$. Then there is a smooth embedding $f$ from $\Re^{0}(G)$ into $\mathbb{R}^{k}$ and $g_{1}, \cdots, g_{k} \in G$ for some $k$, such that $f(\phi)=$ $\left(l\left(\phi\left(g_{1}\right)\right), \cdots, l\left(\phi\left(g_{k}\right)\right)\right)$ for $\phi \in \Re^{0}(G)$.

Proof: A hyperbolic isometry in Iso $\left(H_{\mathrm{H}}^{n}\right)$ which fixes 0 and $\infty$ is of the form:

$$
\left[\begin{array}{ccc}
M & 0 & 0 \\
0 & \cosh r & \sinh r \\
0 & \sinh r & \cosh r
\end{array}\right]
$$

where $C+D j=M \in S p(n-1)$, which can be identified with

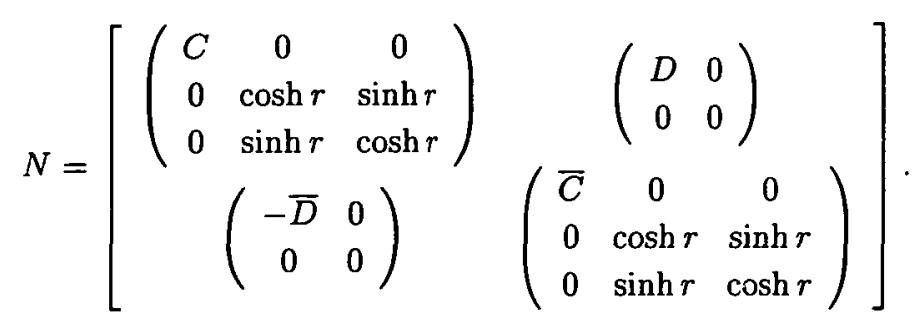


Since

$$
\left[\begin{array}{cc}
C & D \\
-\bar{D} & \bar{C}
\end{array}\right]
$$

is in $U(2 n-2)$, there is a matrix

$$
J=\left[\begin{array}{ll}
E & F \\
G & H
\end{array}\right]
$$

so that

$$
J \cdot\left[\begin{array}{cc}
C & D \\
-\bar{D} & \bar{C}
\end{array}\right] \cdot J^{-1}
$$

is equal to a diagonal matrix $\left(e^{i \theta_{1}}, \cdots, e^{i \theta_{2 n-2}}\right)$. Then

$$
\left[\left(\begin{array}{cc}
E & 0 \\
0 & I \\
G & 0 \\
0 & 0
\end{array}\right)\left(\begin{array}{cc}
F & 0 \\
0 & 0 \\
H & 0 \\
0 & I
\end{array}\right)\right]
$$

conjugates $N$ into the form:

$$
N^{\prime}=\left[\begin{array}{c}
\left(\begin{array}{ccc}
A & 0 & 0 \\
0 & \cosh r & \sinh r \\
0 & \sinh r & \cosh r
\end{array}\right) \\
\left(\begin{array}{ll}
0 & 0 \\
0 & 0
\end{array}\right) \\
\left(\begin{array}{cc}
0 & 0 \\
0 & 0
\end{array}\right) \\
\left.\begin{array}{ccc}
B & 0 & 0 \\
0 & \cosh r & \sinh r \\
0 & \sinh r & \cosh r
\end{array}\right)
\end{array}\right]
$$

where $A, B$ are diagonal matrices. Then $\operatorname{tr} N^{\prime}=\sum \cos \theta_{i}+4 \cosh r$. Now we can conclude the same as in Lemma 2, saying that there is a polynomial relation between $\operatorname{tr} N$ and $\cosh r$. By viewing $\Re^{0}(G)$ as a subset of $\mathbb{C}^{N}$ for some $N$, we can proceed with the proof as in Theorem 2.

It is a well-known fact that the irreducible representation $\phi$, from some group $G$ into $G L_{n}(\mathbb{F})$, is determined up to conjugacy by its character $\chi_{\rho}$, where $\chi_{\rho}(g)=\operatorname{tr}(\rho(g))$. In general, the translation length of an isometry does not determine the trace of the isometry, but in the group of isometries of hyperbolic space, knowing a large enough number of translation lengths of the group is sufficient to determine its character by our theorem. So we get the following.

COROLlaRY 3. Let $R^{0}(G)$ be the space of irreducible representations from a finitely presented group $G$ into Iso $(X)$ where $X$ is a rank one symmetric space of noncompact type other than a Cayley hyperbolic plane. Identify Iso $(X)$ with some linear subgroup in $G L_{n}(\mathbb{F})$, where $\mathbb{F}$ is real or complex. Then there is a finite set of elements, 
$g_{1}, \cdots, g_{N}$ such that if $l\left(\rho\left(g_{i}\right)\right)=l\left(\phi\left(g_{i}\right)\right)$ for $i=1, \cdots, N$, then $\chi_{\rho}=\chi_{\phi}$ for any $\rho, \phi \in R^{0}(G)$.

In [5], it is also shown that

THEOREM $1^{\prime}$. Let $X$ be a rank one symmetric space of non-compact type. Let $\rho, \phi: G \rightarrow$ Iso $(X)$ be two irreducible representations having proportional marked length specta, that is, $l(\rho(g))=c l(\phi(g))$ for some constant $c$. Then $c=1$ and so they are conjugate.

This theorem is plausible for hyperbolic surfaces since if we pinch a closed geodesic, the closed geodesics crossing that closed geodesic transversely tend to get longer. This theorem asserts that it is also true for the higher dimensional case. From this theorem and the same proof as in Theorem 2, we get

CoRollary $3^{\prime}$. Let $R^{0}(G)$ be the space of irreducible representations from a finitely presented group $G$ into Iso $(X)$ where $X$ is a rank one symmetric space of noncompact type other than a Cayley hyperbolic plane. Identify Iso $(X)$ with some linear subgroup in $G L_{n}(\mathbb{F})$ where $\mathbb{F}$ is real or complex. Then there is a finite set of elements, $g_{1}, \cdots, g_{N}$ such that if $l\left(\rho\left(g_{i}\right)\right)=c l\left(\phi\left(g_{i}\right)\right)$ for $i=1, \cdots, N$ and a fixed constant $c$, then $\chi_{\rho}=\chi_{\phi}$ for any $\rho, \phi \in R^{0}(G)$.

From the spectral theoretic point of view, this amounts to saying that a finite set of the marked largest eigenvalues of the matrices in the image of a representation, determines the irreducible representation up to conjugacy.

\section{EXPONENTIAL ALGEBRAIC CONVERGENCE IN THE SPACE OF NON-ELEMENTARY REPRESENTATIONS}

In this section we only consider representations from a surface group into Iso ${ }^{+}\left(H_{\mathbb{R}}^{3}\right)=$ $P S L_{2}(\mathbb{C})=S O_{0}(3,1)$. In the late 70 s Thurston constructed hyperbolic metrics on 3manifolds which fibre over the circle where the monodromy map is pseudo-Anosov. From now on, $\psi$ is a pseudo-Anosov map on a closed hyperbolic surface $S$. First, pick any accumulation point $M_{\psi, Y}$ of $Q\left(\psi^{-i}(X), Y\right)$ in the boundary of the Bers slice $T_{Y}$, where $Q(X, Y)$ is the hyperbolic 3-manifold which has two hyperbolic surfaces $X$ and $Y$ as two ideal boundaries. Then the sequence $\psi^{n}\left(M_{\psi, Y}\right)$ converges to $M_{\psi}$ by Thurston's double limit theorem. Furthermore, $M_{\psi}$ is a fixed point of $\psi$, and there is an isometry $\alpha: M_{\psi} \rightarrow M_{\psi}$ in the homotopy class of $\psi$. Then $M_{\psi} /\langle\alpha\rangle$ is the desired hyperbolic manifold. For the details and background, we refer readers to [6].

In this section we want to show that the convergence $\psi^{n}\left(M_{\psi, Y}\right) \rightarrow M_{\psi}$ is exponentially fast on the basis of McMullen's argument.

DEFINITION 2. We say $\rho_{i} \rightarrow \rho$ exponentially fast if for each $g \in G$, there is a 
$\lambda<1$ such that

$$
\sup _{x \in S_{\infty}^{2}}\left|\rho_{i}(g)(x)-\rho(g)(x)\right|=O\left(\lambda^{i}\right) .
$$

Let $V(S)$ be the space of non-elementary representations from $\pi_{1}(S)$ into Iso ${ }^{+}\left(H_{\mathbf{R}}^{3}\right)$ up to conjugacy in Iso $^{+}\left(H_{\mathbf{R}}^{3}\right)$. Note here that even though a representation is reducible, that is, fixes some hyperbolic 2-plane, any orientation preserving isometry which is an identity on this plane must be an identity, so the conjugacy action is free on the space of non-elementary representations. This implies that $V(S)$ is a smooth manifold, actually a complex manifold when we identify $\operatorname{Iso}^{+}\left(H_{\mathbb{R}}^{3}\right)$ with $P S L_{2}(\mathbb{C})$.

By Corollary 1, there is a smooth embedding of $V(S)$ into $\mathbb{R}^{N}$ for some large $N$, using the smooth functions $[\rho] \rightarrow l_{\rho}(g)$ for some $g_{1}, \cdots, g_{N} \in \pi_{1}(S)$.

On a smooth manifold, there is a natural notion of exponential convergence using a local Euclidean chart. So it is sufficient to check this property in $\mathbb{R}^{N}$ using a translation length chart. The following theorem is due to McMullen [6]. In the proof he used the character space of $V(S)$ to embed it into $\mathbb{C}^{N}$ for some large $N$. We use a translation length coordinate chart to simplify the argument.

Theorem 4. (McMullen) For any pseudo-Anosov mapping $\psi \in \operatorname{Mod}(S)$, and any $Y \in \operatorname{Teich}(\bar{S}), \psi^{i}\left(M_{\psi, Y}\right)$ converges to $M_{\psi}$ exponentially fast.

PRoOF: There is a quasi-isometry $\Psi: M_{\psi, Y} \rightarrow M_{\psi, Y}$ realising $\psi$. Furthermore the injectivity radius of $M_{\psi, Y}$ in its convex core is bounded below and above. By the Geometric inflexibility theorem (see [6, Theorem 2.11]) there are constants $C$ and $D>0$, and a quasi-isometry

$$
\Phi: M_{\psi, Y} \rightarrow M_{\psi, Y}
$$

homotopic to $\Psi$, such that the quasi-isometry constant $L(\Phi, p)$ at a point $p$ in its convex core $K$ satisfies

$$
\log L(\Phi, p) \leqslant C \exp (-D d(p, \partial K)) .
$$

Let $\alpha: M_{\psi} \rightarrow M_{\psi}$ be an isometry realising $\psi$ and $f: S \rightarrow M_{\psi}$ be a marking. Since $\alpha$ acts like a translation along $\mathbb{R}$ when we identify $M_{\psi}$ with $S \times \mathbb{R}$, its translates $\Sigma_{i}=\alpha^{i}(f(S))$ are disjoint. On the other hand there is an asymptotic isometry $h: E \rightarrow E^{\prime}$ between geometrically infinite ends of $M_{\psi}$ and $M_{\psi, Y}$. Let $f_{i}: S \rightarrow M_{\psi, Y}$ be the marking given by the composition

$$
f_{i}=h \circ \alpha^{-i} \circ f .
$$

Then $\psi^{i}\left(M_{\psi, Y}\right)=\left(f_{i}, M_{\psi, Y}\right)$. Pick any non-trivial element $\gamma \in \pi_{1}(S)$. Let $g$ and $g_{i}$ be geodesic representatives in $M_{\psi}$ and $\psi^{i}\left(M_{\psi, Y}\right)$. Then for a sufficiently large $i, d\left(g_{i}, \partial K\right) \geqslant$ $\mathrm{C} i>0$. By this estimate $\Phi$ is a $\left(1+\varepsilon_{i}\right)$-quasi-isometry at $g_{i}$, where

$$
\varepsilon_{i}=O\left(\exp \left(-D d\left(g_{i}, \partial K\right)\right)\right)=O\left(\lambda^{i}\right) .
$$


Then

$$
\left|l_{\psi^{i}\left(M_{\psi, \gamma}\right)}(\gamma)-l_{\psi^{i-1}\left(M_{\psi, \gamma}\right)}(\gamma)\right| \leqslant \varepsilon_{i}=O\left(\lambda^{i}\right) .
$$

This finishes the proof.

\section{REFERENCES}

[1] G. Faltings, 'Real projective structures on Riemann surfaces', Comp. Math. 48 (1983), 223-269.

[2] A. Fathi, F. Laudenbach and V. Poenaru, 'Travaux de Thurston sur les surfaces', Asterisque 66-67 (1979).

[3] R. Gunning, Lectures on vector bundles over Riemann surfaces (Princeton University Press, Princeton, NJ, 1967).

[4] I. Kim, Geometric structures on manifolds and the marked length spectrum, Thesis (University of California, Berkeley, 1996).

[5] I. Kim, Marked length rigidity of rank one symmetric spaces and their product, (submitted).

[6] C.T. McMullen, Renormalization and 3-Manifolds which fiber over the circle, Annals of Math Studies 142 (Princeton University Press, Princeton, NJ, 1996).

Department of Mathematics

University of California at Berkeley

Berkeley CA 94720

United States of America
Current address:

Department of Mathematics

Korea Advanced Institute of Science and Technolog 373-1 Kusong-dong Yusong-ku

Taejon 305-701

Korea

e-mail: inkang@mathx.kaist.ac.kr 